\title{
Resistance of the oyster pathogen Vibrio tasmaniensis LGP32 against grazing by Vannella sp. marine amoeba involves Vsm and CopA virulence factors
}

Etienne Robino, ${ }^{1 \mathrm{II}}$ Aurore C. Poirier, ${ }^{1 \dagger, \text {, }}$

Hajar Amraoui, ${ }^{1}$ Sandra Le Bissonnais, ${ }^{1}$ Angélique Perret, ${ }^{1}$ Carmen Lopez-Joven, ${ }^{1 *}$ Jean-Christophe Auguet, ${ }^{2}$ Tristan P. Rubio, ${ }^{1 \S}$ Chantal Cazevieille, ${ }^{3}$ Jean-Luc Rolland, ${ }^{1}$ Yann Héchard, ${ }^{4}$ Delphine Destoumieux-Garzón ${ }^{1}$ and Guillaume M. Charrière (1) ${ }^{1 *}$

${ }^{1}$ IHPE UMR 5244, CNRS, Ifremer, University of Montpellier, University of Perpignan Via Domitia, Montpellier, France.

${ }^{2}$ MARBEC, University of Montpellier, CNRS, Ifremer, IRD, Montpellier, France.

${ }^{3}$ INM, University of Montpellier, Montpellier, France. ${ }^{4}$ EBI UMR CNRS 7267, University of Poitiers, Poitiers, France.

\section{Summary}

Vibrios are ubiquitous in marine environments and opportunistically colonize a broad range of hosts. Strains of Vibrio tasmaniensis present in oyster farms can thrive in oysters during juvenile mortality events and behave as facultative intracellular pathogen of oyster haemocytes. Herein, we wondered whether $V$. tasmaniensis LGP32 resistance to phagocytosis is specific to oyster immune cells or contributes to resistance to other phagocytes, like marine amoebae. To address this question, we developed an integrative study, from the first description of amoeba diversity in oyster farms to the characterization of LGP32 interactions with amoebae. An isolate of the Vannella genus, Vannella sp. AP1411, which was collected from oyster farms, is ubiquitous, and belongs to one clade of Vannella that could be found associated with Vibrionaceae. LGP32 was shown to be resistant to grazing by Vannella sp. AP1411 and this phenotype depends on some

Received 20 May, 2019; accepted 4 August, 2019. *For correspondence. E-mail guillaume.charriere@ umontpellier.fr; Tel. +33(0) 4-67-14-46-25. Present addresses: 'Department of School of Veterinary Medicine, University of Surrey, Guildford, UK; "Universidad Austral de Chile, Valdivia, Chile; ${ }^{\S}$ MMSB UMR 5086, University of Lyon, Lyon, France. "The authors contributed equally to this work. previously identified virulence factors: secreted metalloprotease Vsm and copper efflux p-ATPase CopA, which act at different steps during amoeba-vibrio interactions, whereas some other virulence factors were not involved. Altogether, our work indicates that some virulence factors can be involved in multi-host interactions of $V$. tasmaniensis ranging from protozoans to metazoans, potentially favouring their opportunistic behaviour.

\section{Introduction}

Vibrios are $\gamma$-proteobacteria living in aquatic environments ranging from saline to freshwater. They are ubiquitous in marine coastal environments and have evolved the capacity to colonize a broad range of hosts from protozoans to metazoans (Austin, 2010). Vibrios belong to the microbiota of healthy oysters but some species that behave as opportunistic pathogens can thrive in host tissues and cause disease (Lemire et al., 2014; Bruto et al., 2017; Rubio et al., 2019). This occurs mostly as a result of environmental changes such as shifts in water temperature, exposure to high animal densities and stressful farming practices or upon immune suppression of host defences by other microorganisms such as the OsHV-1 virus (de Lorgeril et al., 2018). Currently, vibrioses are recognized as a major factor limiting the development of aquaculture (Le Roux et al., 2015). In addition, vibrios can cause severe disease outbreaks in human populations, the best-known example being cholera. As they are multi-host pathogens, the ecology of vibrios depends on a series of biotic interactions in the environment that influence their ecology, their evolution and their transmission to animal and human hosts (Constantin de Magny et al., 2008; Takemura et al., 2014).

Bacteriovorus protists, such as free-living amoebae (FLA), are bacterial predators present in natural environments. They can be found in most aquatic environments and their predation through grazing activity shapes bacterial communities (Pernthaler et al., 2005). However, it has been shown that some bacteria are able to resist grazing, persist and even grow within amoebae (Greub and 


\section{E. Robino et al.}

Raoult, 2004). The best-known and described example is the facultative intracellular pathogen Legionella pneumophila (Rowbotham, 1980). Predation activity by protists shares cellular and molecular processes with the antibacterial responses of metazoan immune cells, in particular phagocytosis (Boulais et al., 2010). Hence, amoebae have been hypothesized to act as 'evolutionary training ground' for grazing-resistant bacteria, especially for intracellular bacterial pathogens, by favouring the selection of virulence factors (Molmeret et al., 2005).

A number of studies have demonstrated that some Vibrio species adopt intracellular stages (Rosenberg and Falkovitz, 2004; Ma et al., 2009; Vidal-Dupiol et al., 2011; Ritchie et al., 2012; de Souza Santos and Orth, 2014). In particular, intracellular stages are required for strains of Vibrio tasmaniensis to express virulence in oysters (Duperthuy et al., 2011; Rubio et al., 2019). Virulent strains of this species have been isolated from moribund oysters during juvenile mortality events on the French Atlantic coast (Gay et al., 2004; Lemire et al., 2014; Bruto et al., 2017). This species was also found in oyster flesh during juvenile mortalities, as well as in the water column and the sediment of oyster farms in the Mediterranean Thau Lagoon, France (de Lorgeril et al., 2018; Lopez-Joven et al., 2018). Some of the V. tasmaniensis LGP32 virulence factors have been described in oysters. They include secreted proteases, such as Vsm (Binesse et al., 2008) or Vsp (Vanhove et al., 2014), antioxidants such as the superoxide dismutase SodA, efflux pumps to detoxify heavy metals such as the copper p-ATPase CopA (Vanhove et al., 2016), and a type $\mathrm{VI}$ secretion system that mediates the intracellular secretion of toxic effectors (Rubio et al., 2019).

Here, we wondered whether some virulence factors of the Vibrio tasmaniensis LGP32 playing a key role in its interaction with oysters could also be beneficial during other biotic interactions in the environment. With this objective, we first investigated the natural associations between vibrios and marine amoebae. Second, we did comparative cellular biology to study the interaction between LGP32 and an environmental free-living marine amoeba (Vannella sp. strain AP1411) ubiquitous in oyster farms, which belongs to a clade of Vannella $s p$. that establish interactions with vibrios. By performing a combination of flow cytometry, confocal and electron microscopy analyses, we show that LGP32 resists to the predation by Vannella sp. AP1411. Using mutant strains inactivated for previously characterized virulence factors in oysters, we found that the copper p-ATPase efflux pump CopA and the secreted metalloprotease Vsm are both involved. CopA appears to play a role in the intracellular survival of LGP32 whereas Vsm appears to play a role in the inhibition of amoebae motility during grazing.
Altogether, our results indicate that some of the previously identified virulence factors involved in $V$. tasmaniensis LGP32 interaction with oysters, are also involved in resistance to predation by marine amoeba Vannella sp. AP1411, thus potentially favouring opportunism.

\section{Results}

Marine amoebae belonging to the Vannellidae family and present in oyster farming area can interact with vibrios

Although amoebae represent an important category of benthic bacterial grazers, their diversity remains poorly explored in marine environments, particularly in Mediterranean lagoons exploited for oyster farming. Therefore, we first attempt to identify and isolate FLA in marine environments hosting oyster farms. Amoebae were isolated from the Thau lagoon at the vicinity of an oyster farming area. Samples were collected from the water column, oyster gills and sediment at all seasons between the year 2014 and 2015, and at least two cultures of amoebae per fraction and per season were analysed. Cloning-sequencing of the v7 hypervariable region of $18 \mathrm{~S}$ rDNA gene was used to identify the isolated amoebae. A total of 110 sequences were analysed. From 24 different cultures, 103 amoebae sequences showed more than $90 \%$ sequence homology to the Vannella genus, which belongs to the Vannellidae family. The 103 sequences were classified into 11 different Operational Taxonomic Units (OTUs) (Fig. 1 and Table S1). In parallel, $16 \mathrm{~S}$ barcoding of the different amoebae cultures confirmed that only species belonging to the Vannella genus were present in the samples (Fig. S1). One OTU of Vannella sp. was ubiquitous, as it was found in the three fractions during four seasons, and referred to the isolate AP1411 (arrow in Fig. 1). In an attempt to determine whether marine amoebae could be found associated with Vibrionaceae in the environment, a dedicated sampling was performed in 2017 and amoebae were clonally isolated from oyster gills and from water column in another site (Banyuls-sur-mer, France) as an out-group. Then 16s barcoding analyses were performed to characterize the bacteria that remained associated to these Vannella clones 4 weeks after culturing. From all the clones analysed, four were found associated with Vibrionaceae OTUs (Fig. 2A). In order to validate this association, the presence of Vibrionaceae associated with the four Vannella sp. clones was then assessed by FISH using a 16 s probe specific for vibrios (Huggett et al., 2008) (Fig. 2B). Among the four clones that were analysed, two showed an association with Vibrionaceae (0517-TOEc-2 and 0517-TOEc-4). The vibrios could be found inside amoebae as well as outside. These results suggest that amoebae of the Vannella genus can establish interactions with vibrios in the environment. 


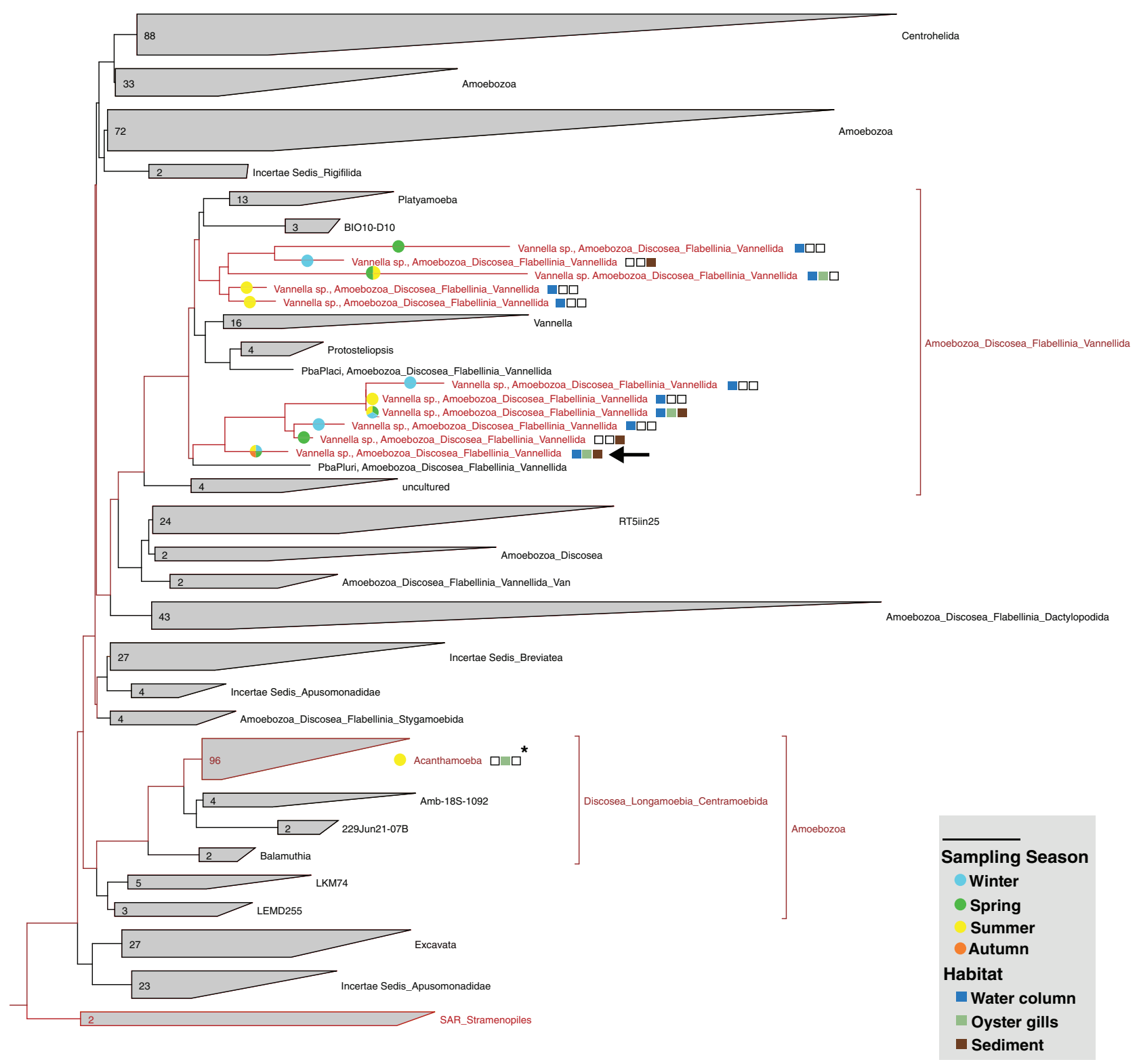

Fig. 1. Most of the isolated free-living amoebae from the Thau lagoon belong to Vannellidae family. Phylogenetic 18S rRNA-based trees of the Amoebozoa constructed with ARB software (Ludwig et al., 2004; http://www.arb-home.de) loaded with the silva database (http://www.arb-silva. de). A base frequency filter was applied using the parsimony quick add marked tool implemented in ARB. Our isolates are written in red. Most of the isolated amoebae during the environmental survey from water column (blue squares) and/or the sediment (brown squares), and/or oyster gills (light green squares) during winter (light blue circles) and/or spring (green circles) and/or summer (yellow circles) and/or autumn (orange circles) belong to the Vannellidae family.

The pathogen $\mathrm{V}$. tasmaniensis LGP32 resists grazing by Vannella sp. AP1411

To study further the interaction between vibrios and Vannella amoebae, we chose the Vannella sp. AP1411 clone, which belongs to the most ubiquitous OTU of marine amoebae and which is not already associated with other vibrios, as shown by FISH (Fig. 2B). The trophozoite, planktonic and cystic forms of Vannella sp. AP1411 were typical of the Vannella genera and very similar to Vannella plurinucleolus (Smirnov et al., 2007), which was also the closest 18S matching sequence for this isolate $(98 \%$ BLASTn score). Transmission electron microscopy of trophozoïtes revealed a typical intracellular organization of Vannella with a well-defined, thick plasma membrane, one nucleus (sometimes two or three), numerous mitochondria and a rich vacuolar system with large digestive vacuoles containing remnants of degraded bacteria, and some phagosomes containing individual bacteria (Fig. S2). 
A

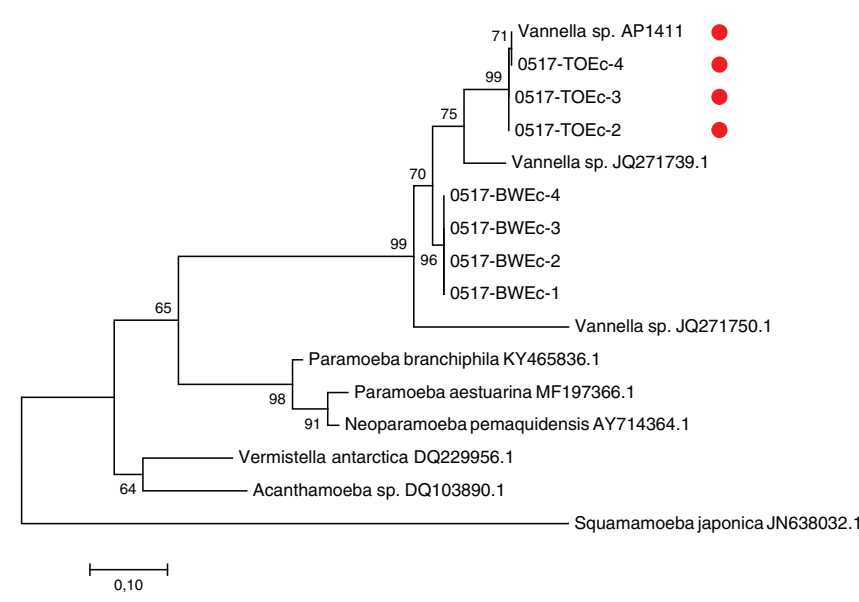

B
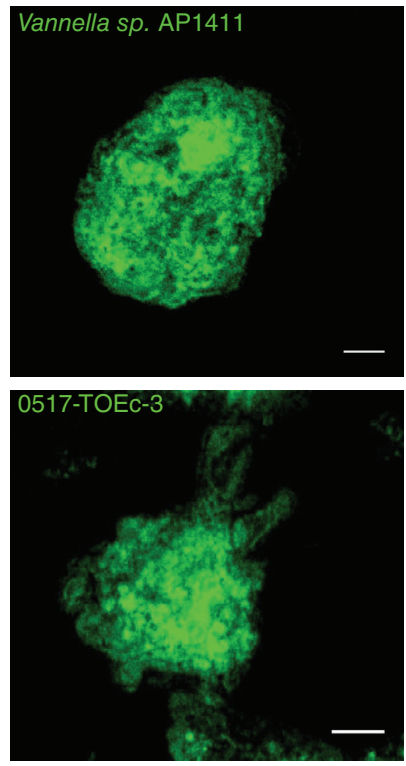

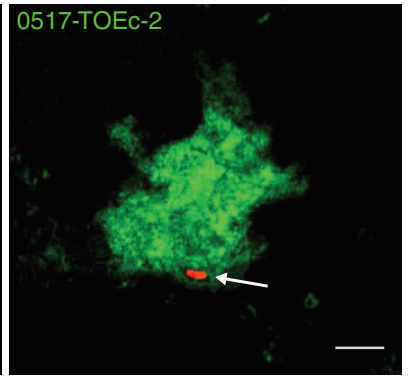

0517-TOEC-4

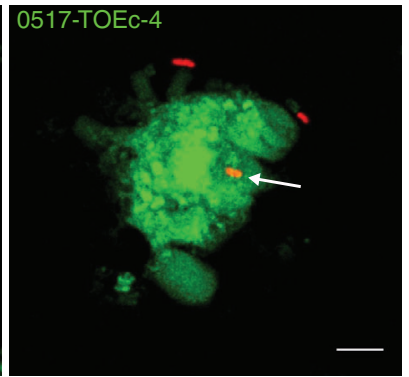

Fig. 2. Amoebae belonging to the Vannella genus can be found associated with Vibrionaceae bacteria in oyster farms. Vannella were clonally isolated from oyster gills from Thau lagoon (Vannella sp. AP1411, 0517-TOEc-2, 0517-TOEc-3, and 0517-TOEc-4) or from seawater in Banyuls-surMer (0517-BWEc-1, 0517-BWEc-2, 0517-BWEc-3, and 0517-BWEc-4), then 16s barcoding sequencing was performed to identify potentially associated Vibrionaceae.

A, The 18S rRNA tree was constructed with maximum-likelihood by bootstrap method (1000 replications), isolates found associated with Vibrionaceae by $16 \mathrm{~s}$ barcoding analyses are indicated with red dots.

B, Vibrionaceae were revealed by FISH using the VIB572a Vibrionaceae specific probe (Red) and amoebae cells were counterstained with FITC. Vibrios could be observed both outside and inside (arrows) amoebae by confocal microscopy. Scale bar: $5 \mu \mathrm{m}$.

To study LGP32 - Vannella sp. AP1411 interactions, grazing assays were performed on solid media (nonnutrient seawater agar). Vibrio strains (pathogenic or non-pathogenic to oysters) that constitutively express the Green Fluorescent Protein (GFP) were used to seed the plates and monitor the abundance of living vibrios by fluorescence quantification. In parallel, Vannella sp. AP1411 growth was monitored by counting the cell density at the surface of the grazing lawns. When the amoeba was cultured in the presence of the non-virulent Vibrio tasmaniensis $\mathrm{LMG} 0012^{\top}$, a rapid decay of GFPexpressing bacteria was observed 4 days after the beginning of the experiment, whereas in the case of the pathogenic V. tasmaniensis LGP32, the amount of GFPexpressing bacteria remained stable for up to 7 days (Fig. 3A). Amoeba growth (cells count) on the vibrio lawns counter mirrored the kinetics of the decay of GFPexpressing bacteria. Indeed, amoeba density increased much faster in the presence of LMG20012 ${ }^{\top}$ than in the presence of LGP32 (Fig. 3B). These data indicate that Vannella sp. AP1411 do not prey efficiently on the virulent LGP32, whereas they can feed on the non-virulent LMG20012'.

To determine whether the amoebal growth defect observed in the presence of LGP32 was due to inefficient phagocytosis and/or resistance to intracellular degradation by LGP32, we next quantified the capacity of Vannella sp. AP1411 to phagocytose LGP32 and LMG20012 ${ }^{\top}$, using flow cytometry (Fig. S3). The amount of amoebae carrying GFP-vibrios was quantified 3 and 6 days after grazing, at 3 days after the beginning of the interaction, $100 \%$ of amoebae were found to carry GFP-LMG20012 ${ }^{\top}$ or GFP-LGP32 (Fig. 3C). However, at day 6, the percentage of amoebae carrying GFP-LMG20012 ${ }^{\top}$ decreased down to $20 \%$ whereas it remained stable with GFP-LGP32 (Fig. 3C) suggesting that LGP32 could resist intracellular degradation. Confocal microscopy investigations confirmed that the amoebae were able to phagocytose both vibrios (Fig. 3D). These observations also confirmed that 6 days after the onset of the grazing assay, $80 \%$ of amoebae were devoid of any GFP-LMG20012 ${ }^{\top}$ as quantified by flow cytometry, with only $20 \%$ of amoebae that were still carrying intracellular GFP-LMG20012 ${ }^{\top}$, whereas $100 \%$ of the amoebae were still carrying numerous GFP-LGP32 (Fig. 3D). This suggests that GFP-LGP32 is able to resist intracellular degradation after engulfment in contrary to the non-virulent control strain GFP-LMG20012 ${ }^{\top}$ that seemed to be digested by the amoeba.

To investigate further the intracellular fate of the vibrios inside amoebae, transmission electron microscopy was performed 3 days after the onset of grazing (Fig. 3E). Most LMG20012 ${ }^{\top}$ cells were found clustered inside vacuoles and showed altered morphologies whereas LGP32 remained intact inside amoebae and were mostly found in 
individualized phagosomes confirming that LGP32 is resistant to intracellular degradation compared with LMG20012' Altogether, these results show that LGP32 is resistant to grazing by Vannella sp. AP1411 as opposed to the nonvirulent LMG20012 ${ }^{\top}$, and that LGP32 is able to resist to intracellular degradation by the amoeba.
A

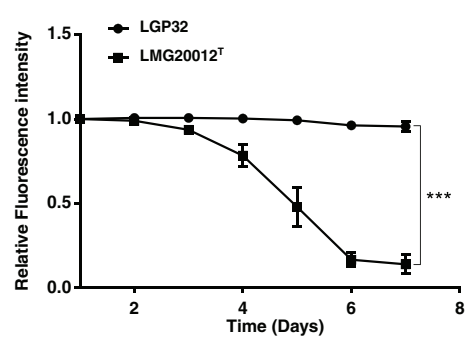

D
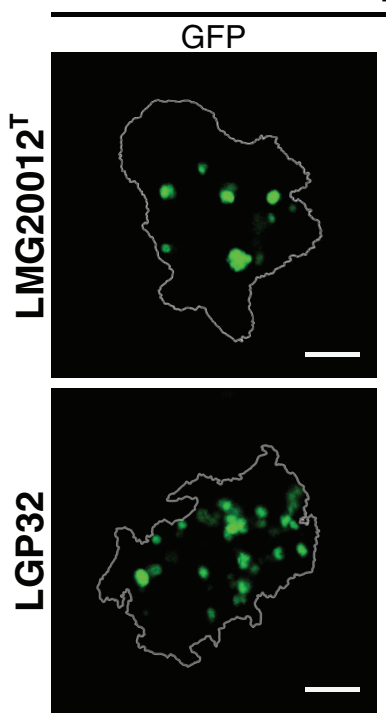

Day 3
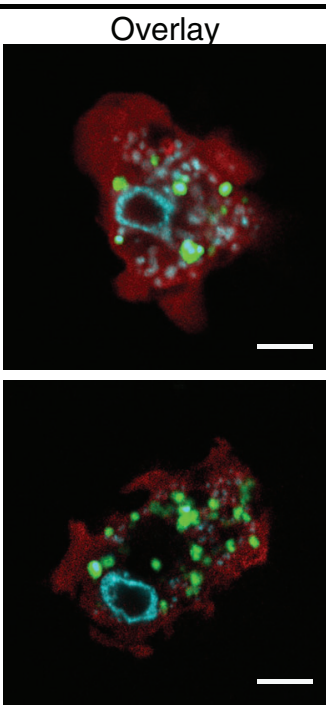

E

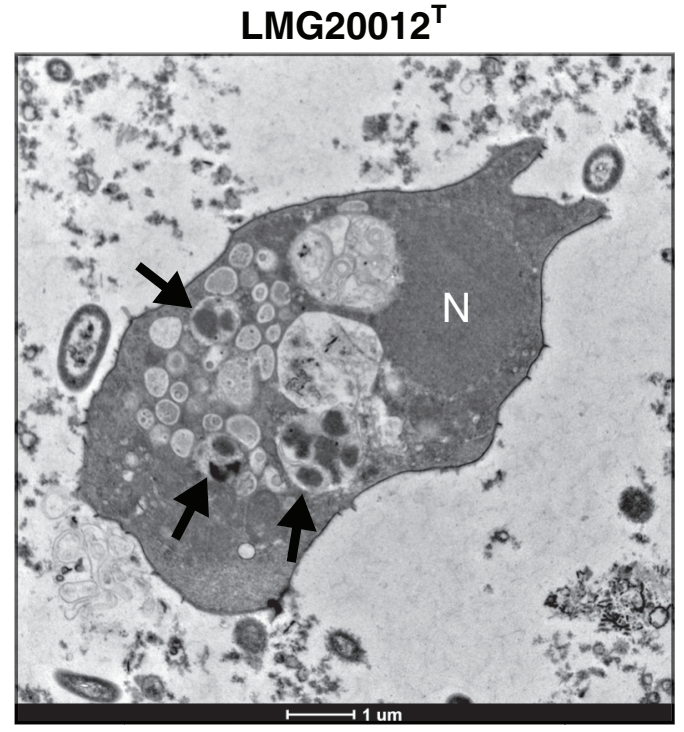

C
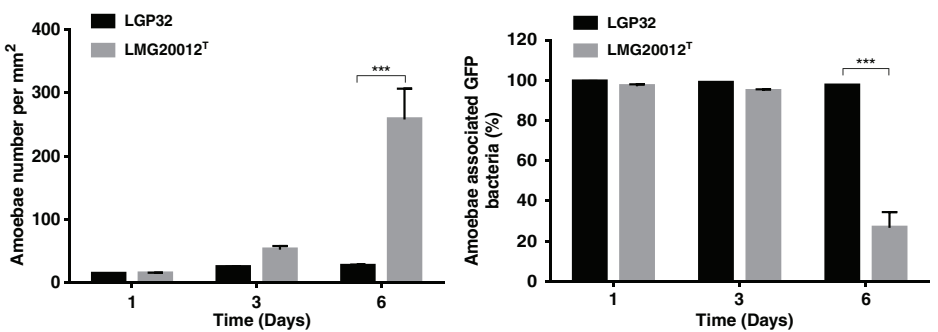

Day 6
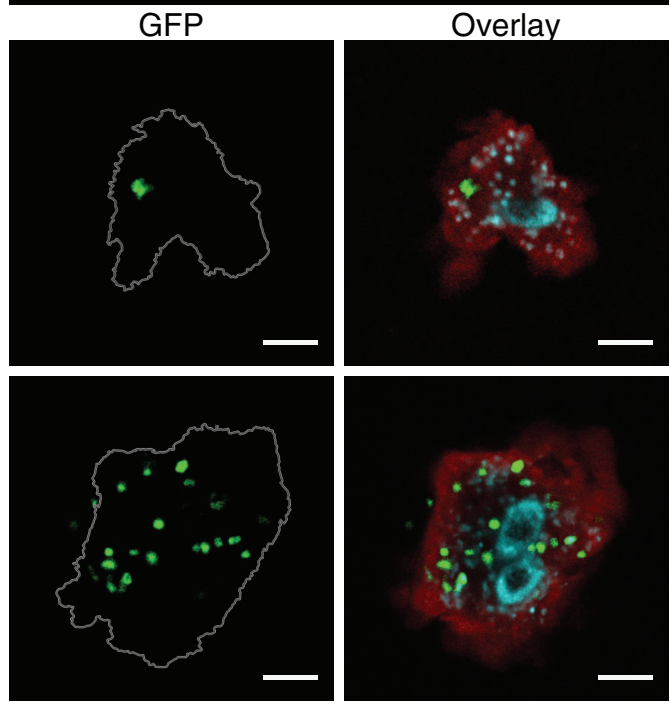

LGP32

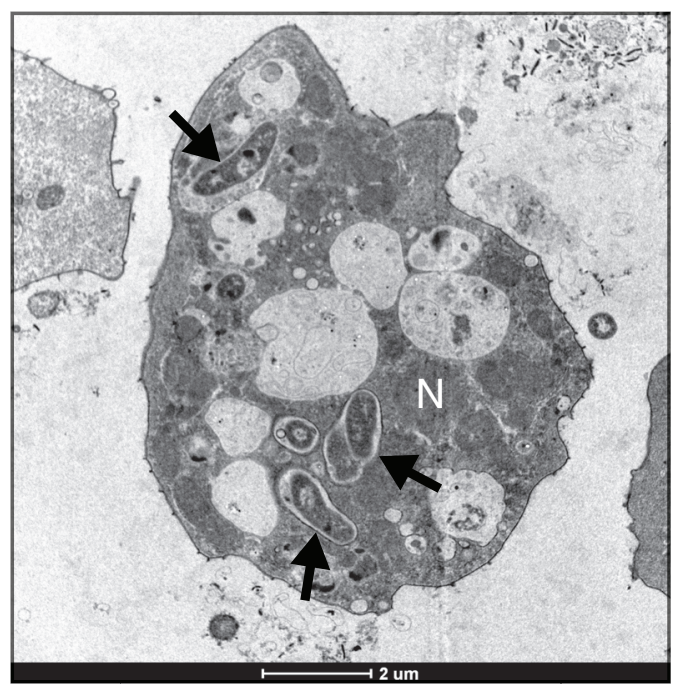


A

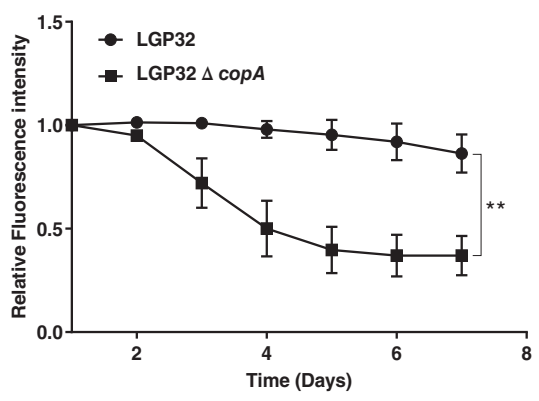

D

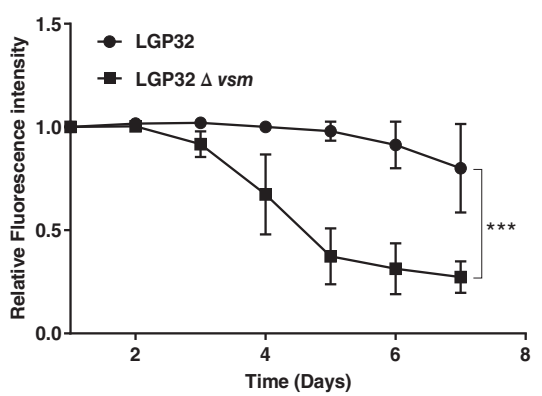

B

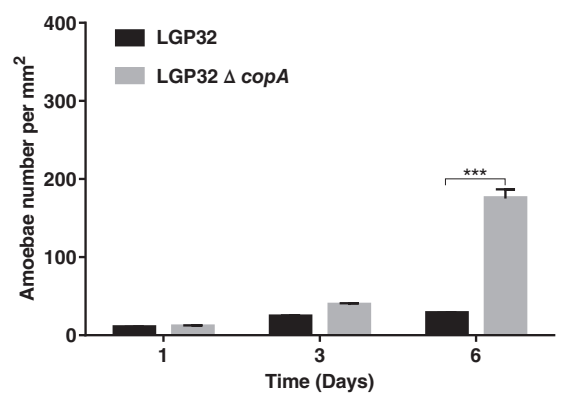

E

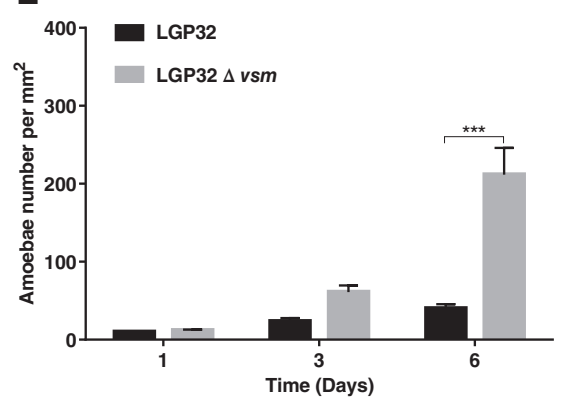

C

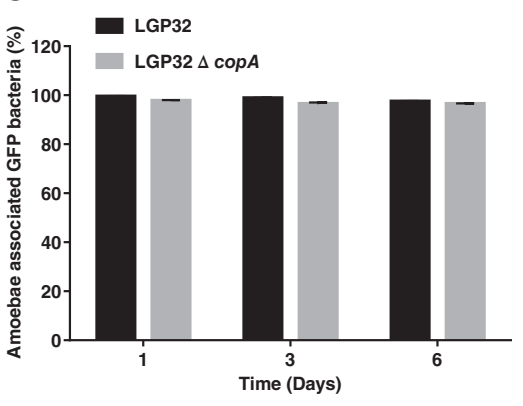

$\mathbf{F}$

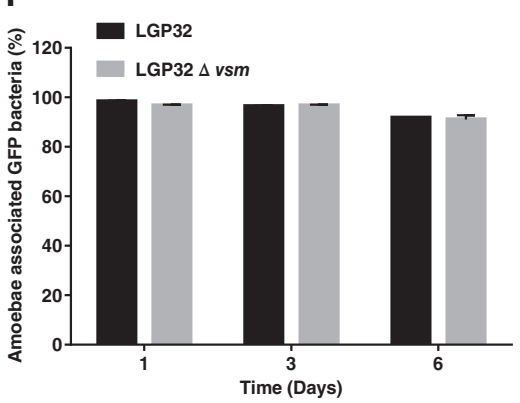

Fig. 4. Copper p-ATPase efflux pump CopA and metalloprotease Vsm are involved in LGP32 resistance to grazing by Vannella sp. 1411. A-D, Bacterial resistance of $\Delta$ copA and $\Delta v s m$ strains of LGP32 was assessed by measurement the fluorescence of the GFP-expressing bacteria, after contact with amoebae. Each condition was performed in triplicate and the results shown are the average of three independent experiments. Values are presented \pm SEM, ${ }^{\star \star} P<0.01,{ }^{\star \star \star} P<0.001$ (RM-ANOVA).

$\mathrm{B}-\mathrm{E}$, Amoeba growth was monitored by manual counting under phase light microscopy. Each condition was counted in three technical replicates. The results shown are representative of two independent experiments \pm SEM. Data were analysed by two-way ANOVA with Holm-Sidak's multiple comparisons test, ${ }^{* *} P<0.001$.

$\mathrm{C}-\mathrm{F}$, In two independent experiments, percentage of amoebae associated with GFP bacteria was measured by flow cytometry. Each condition was performed in three technical replicates. The results shown are representative of two independent experiments \pm SEM. Data were analysed by two-way ANOVA with Holm-Sidak's multiple comparisons test.

\section{The Vsm and CopA virulence factors participate in LGP32 resistance to grazing by marine amoeba}

To investigate further the molecular basis of LGP32 resistance to predation by Vannella sp. AP1411, we studied the phenotype of mutant strains of LGP32 deleted for virulence factors previously identified to be involved in its interaction with oysters. On the one hand, we examined stress resistance systems, that are, the antioxidant SodA, and the copper resistance systems CopA and CusAB, involved in intraphagosomal resistance to antimicrobial activities of oyster immune cells (Vanhove et al., 2016) and on the other hand, we examined secreted virulence factors, that are, the metalloprotease Vsm and the serine protease Vsp that both play a role in oyster pathogenesis (Binesse et al., 2008; Vanhove et al., 2014); as well as the metalloprotease InhA (the orthologue of PrtV) that has been involved in multiple biotic interactions of vibrios (Vaitkevicius et al., 2006), and finally the T6SS1 secretion system required for cytotoxicity towards oyster immune cells (Rubio et al., 2019). Among the eight mutant strains of LGP32 tested here, only

Fig. 3. LGP32 is resistant to grazing by marine amoebae Vannella sp. AP1411.

Grazing assays were performed with Vannella sp. AP1411 with GFP-expressing strains of LGP32 or of the avirulent LMG20012'.

$A$, Bacteria survival was assessed by monitoring the intensity of the GFP fluorescence. Each condition was performed in triplicate and the results represent the average of three independent experiments. Values are presented \pm SEM ${ }^{* \star *} P<0.001$ (RM-ANOVA).

$\mathrm{B}$, Amoebae growth was monitored by manual counting under phase light microscopy. Each condition was performed in three technical replicates and depicted results are representative of two independent experiments \pm SEM. Data were analysed by two-way ANOVA with Holm-Sidak's multiple comparisons test, ${ }^{\star \star \star} P<0.001$.

$\mathrm{C}$, The percentage of amoebae carrying GFP-expressing bacteria was quantified by flow cytometry. Each condition was performed in three technical replicates and depicted results are representative of two independent experiments \pm SEM. Data were analysed by two-way ANOVA with Holm-Sidak's multiple comparisons test, ${ }^{* *} P<0.001$.

D, LMG20012 ${ }^{\top}$ and LGP32 were observed inside amoebae by confocal microscopy 3 days and 6 days after contact. Nuclei were stained with DAPI (Blue) and amoeba proteins with Blue Evans (Red). Scale bar: $5 \mu \mathrm{m}$.

E, Transmission electron microscopy was performed on amoebae, 3 days after grazing on LGP32 or LMG20012 ${ }^{\top}$ lawns. Avirulent LMG20012 ${ }^{\top}$ vibrios were found clustered in intracellular vacuoles with an altered shape (arrows), compared with extracellular vibrios around the amoeba. Virulent LGP32 vibrios were found mostly in individualized phagosomes, and appeared intact (arrows). Scale bar: 1 and $2 \mu \mathrm{m}$. 
the $\Delta$ copA and $\Delta v s m$ deletion mutants were significantly less resistant to grazing by Vannella sp. AP1411 than the wild-type strain, as shown by a faster clearance of GFP.

\section{A}
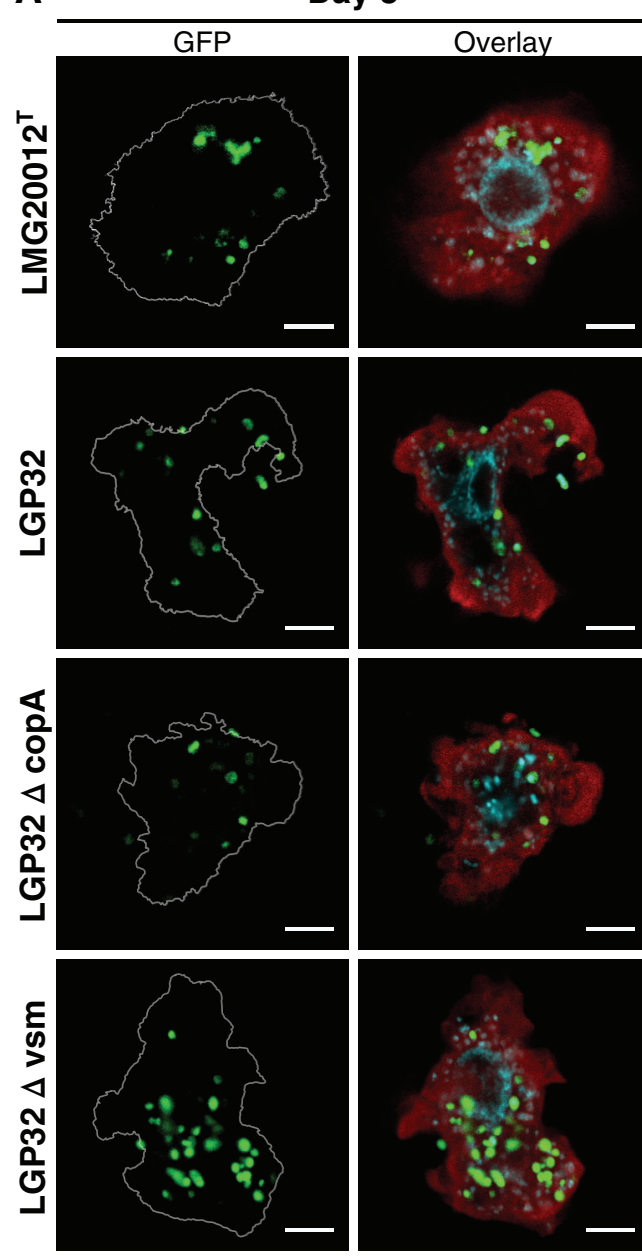

B
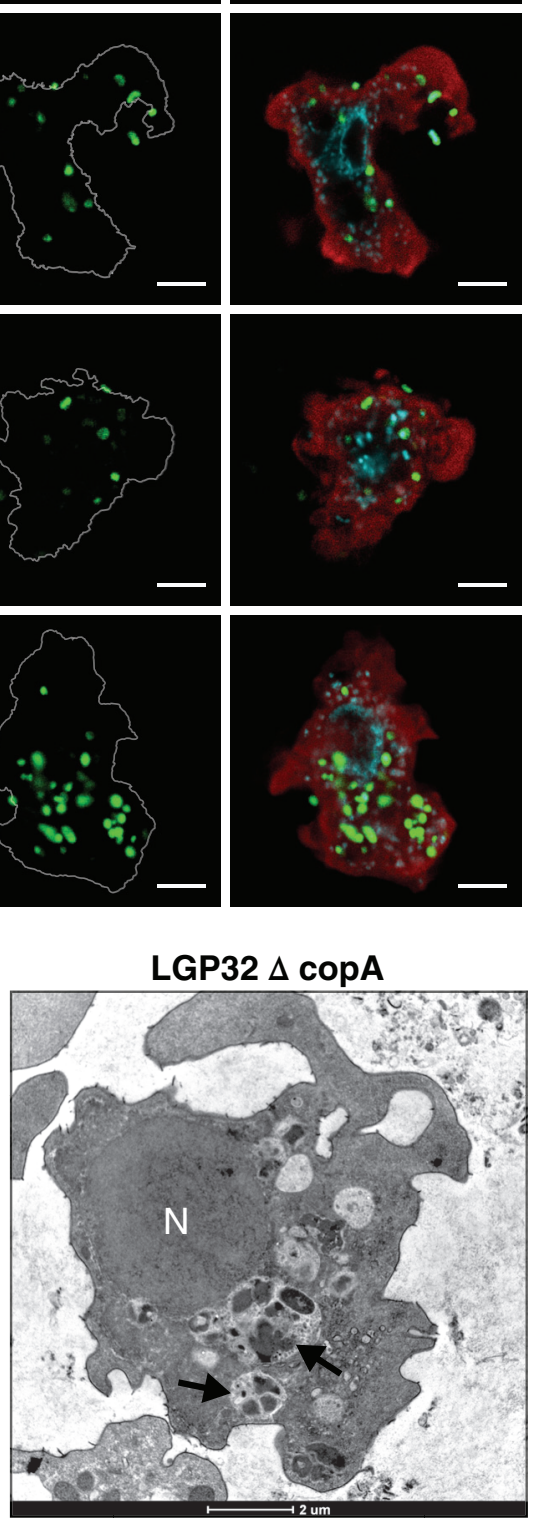

vibrios on grazing lawns (Fig. 4A and D; and Fig. S4). Accordingly, on lawns of both $\Delta$ copA and $\Delta v s m$, rapid bacterial clearance correlated with faster amoebal growth as
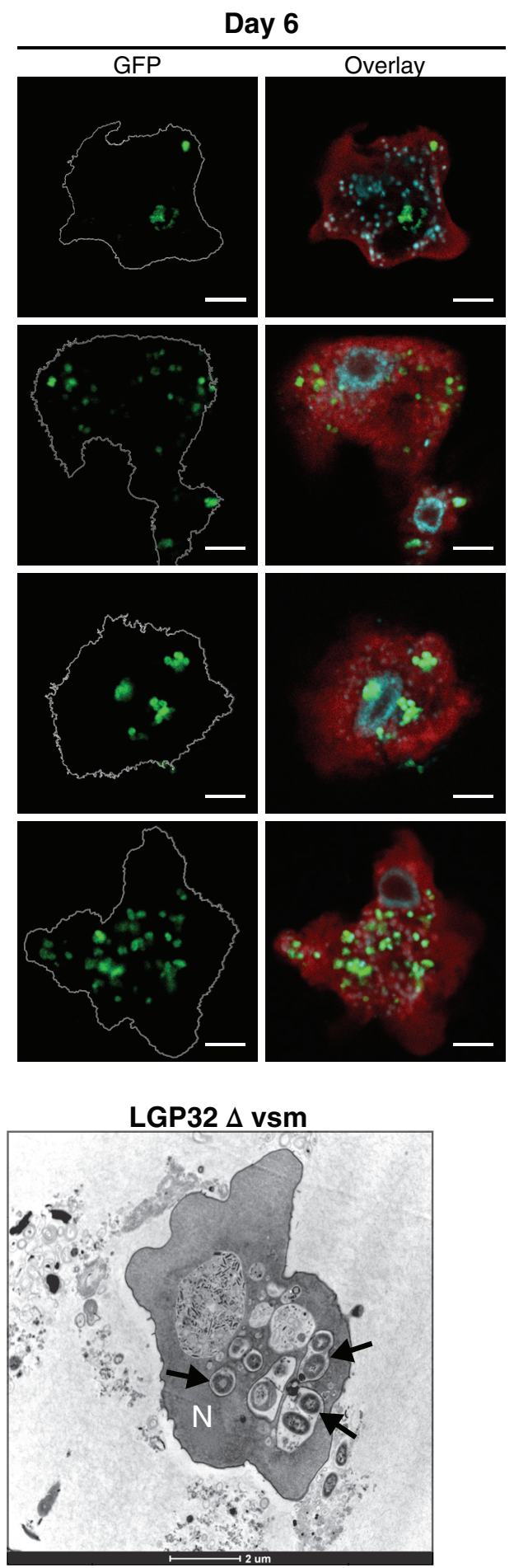

Fig. 5. Copper p-ATPase efflux pump CopA is necessary to resist to intracellular degradation in amoeba whereas the metalloprotease Vsm is not. A, Intracellular vibrios were observed 3 days and 6 days after infection, by confocal microscopy. Nuclei were stained with DAPI and amoeba proteins with Blue Evans. Scale bar: $5 \mu \mathrm{m}$.

$\mathrm{B}$, Transmission electron microscopy was performed on amoeba 3 days after grazing on $\triangle \operatorname{cop} A$ or $\Delta v s m$ mutant strains. Mutant $\Delta$ copA vibrios were found clustered in intracellular vacuoles with an altered shape and appearance (arrows). Vibrios $\Delta$ vsm LGP32 were found mostly in individualized phagosomes, and appeared intact (arrows), scale bar: $2 \mu \mathrm{m}$. 

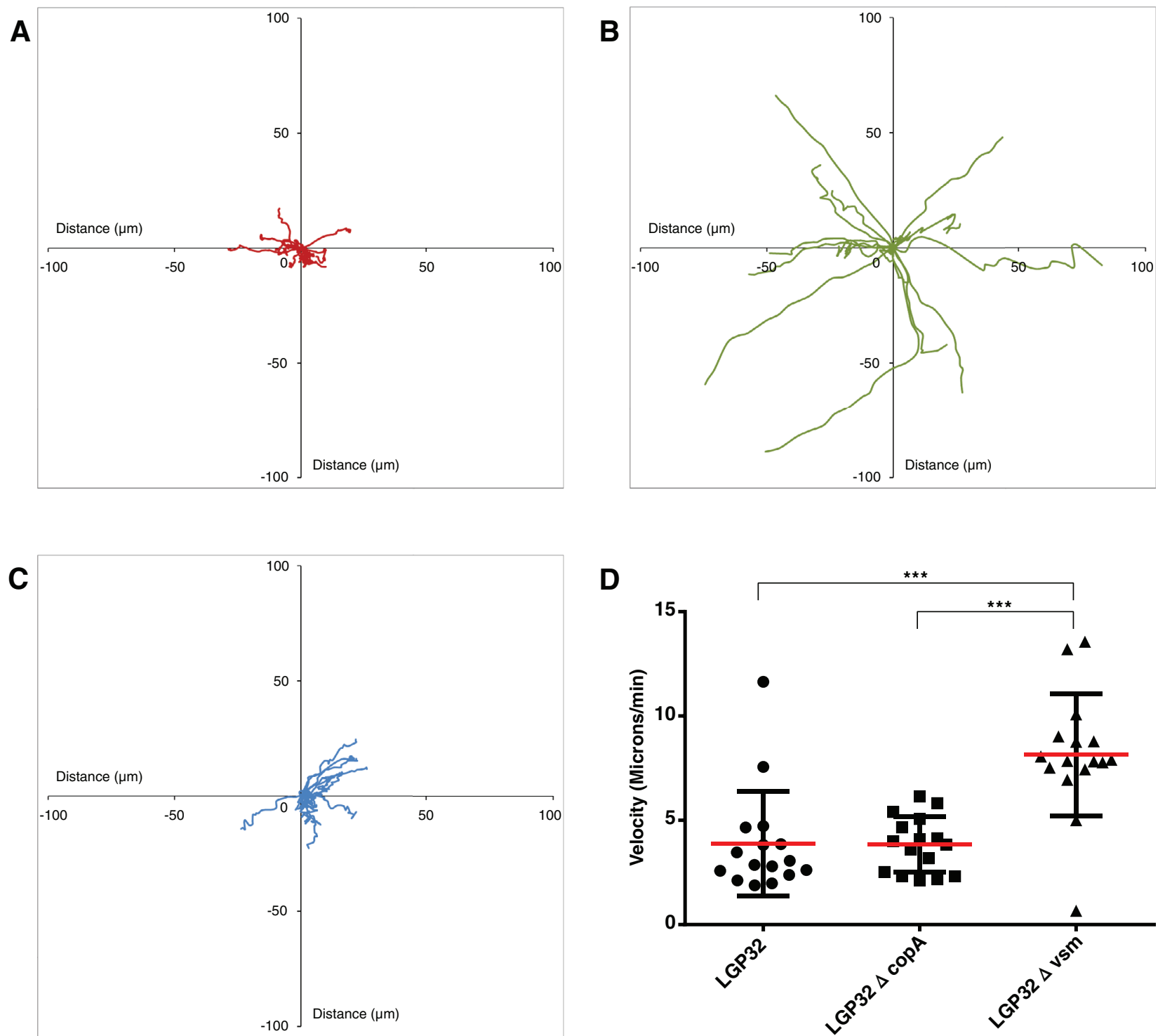

Fig. 6. Extracellular metalloprotease Vsm decreases the velocity of the amoebae Vannella sp. AP1411 and alters amoeba grazing efficiency. Amoebae migration on LGP32wt (A), $\Delta v s m$ (B) or $\Delta \operatorname{copA}(\mathrm{C})$ mutant strains lawns was recorded by time-lapse phase light microscopy during $10 \mathrm{~min}$. Each presented track corresponds to the migration of one amoeba. The results shown are representative of at least two independent experiments. (D) Average velocity of amoebae on LGP32wt, $\Delta$ copA or $\Delta v s m$ mutant strains. The results are representative of at least two independent experiments \pm SD. Data were analysed by Kruskal-Wallis test with Dunn's multiple comparisons test, ${ }^{\star \star \star} P<0.001$.

shown by the higher amoeba density than on wild-type LGP32 lawns (Fig. 4B and E). These results show that both the CopA resistance factor and the Vsm virulence factor play a role in the resistance of LGP32 to grazing by Vannella sp. AP1411 whereas the other tested virulence factors did not seem to be involved.

The p-ATPase CopA confers resistance to intracellular killing and the extracellular protease Vsm inhibits amoebae motility during grazing

To investigate further the role of LGP32 virulence factors in resistance to amoeba grazing, we analysed different steps of the grazing process, from phagocytosis to intracellular degradation. Flow cytometry analyses showed that the percentage of phagocytosis (Fig. 4C and F) and the phagocytosis index (Fig. S5) were not significantly different between the mutant strains $\triangle \mathrm{cop} A$ and $\Delta v s m$ and the wild-type LGP32, which suggested that the efficiency of the phagocytosis process was not disturbed by CopA or Vsm. Confocal and TEM microscopy confirmed that both mutant strains were readily phagocytosed by the amoeba, and revealed that $\triangle$ copA LGP32 vibrios were found mostly clustered in vacuoles and the bacteria appeared to have an altered morphology after 3 days of grazing. These observations suggest that $\triangle$ copA LGP32 
was more sensitive to intracellular degradation, indicating a role for $\operatorname{copA}$ in resistance of LGP32 to intracellular degradation. In contrast, $\Delta v s m$ LGP32 appeared intact inside vacuoles and individual phagosomes in a similar manner than the wild-type strain of LGP32, suggesting that Vsm is not involved in LGP32 resistance to intracellular degradation (Fig. 5A and B).

To investigate further the role of Vsm in LGP32 resistance against grazing by Vannella sp. AP1411, amoeba grazing was recorded by time-lapse microscopy. Amoebae grazing behaviour on vibrio lawns was analysed using $\Delta v s m$ LGP32 and $\Delta$ copA LGP32 lawns and compared with wild-type strain. Cell tracking was performed to measure amoeba migratory speed on the different lawns of vibrios (Fig. 6) and the most striking difference that could be observed was that amoebae migratory speed was two fold faster $(p<0.001)$ on lawns of $\Delta v s m$ LGP32 compared with wild-type or $\Delta$ copA LGP32 lawns (Supplementary Movies 1,2 and 3), with mean velocities of 8.5, 3.9 and $3.8 \mu \mathrm{m} \mathrm{min}^{-1}$ for $\Delta v s m$ LGP32, wild-type LGP32 and $\Delta$ copA LGP32 respectively (Fig. 6D). These results indicate that the metalloprotease Vsm acts extracellularly by inhibiting amoeba motility, which likely disturbs grazing efficiency of amoeba, whereas CopA participate to the intracellular survival capacity of LGP32.

\section{Discussion}

In this study, we uncovered that the oyster pathogen V. tasmaniensis LGP32 is resistant to grazing and intracellular degradation by free-living marine amoebae of the Vannella genus. Here we used Vannella sp. AP1411 as a representative amoeba isolate, which belongs to the genus of amoeba that is ubiquitous in the oyster farming environment and that interacts with vibrios in the environment. We also uncovered some of the molecular determinant that are involved in the resistance of LGP32 to Vannella sp. AP1411, the copper efflux p-ATPase CopA and the secreted metalloprotease Vsm. These results indicate that at least two different mechanisms contributing to $V$. tasmaniensis LGP32 pathogenicity in oysters are also involved in resistance to amoeba grazing.

Amoebae of the Vannellidae family were found to be ubiquitous in the Thau lagoon, a Mediterranean coastal lagoon used for oyster farming ( $20 \%$ of its total surface). Indeed, most of the isolated amoebae branched into the Vannella genus, independently of seasons. Together with the present study, the current literature suggests that Vannellidae amoebae are frequent in aqueous environments, mostly in saltwater but are also frequently observed in freshwater (Smirnov et al., 2007). By performing $16 \mathrm{~s}$ barcoding sequencing and FISH analysis, we were able to find that some Vannella sp. isolated from oyster farms were associated with vibrionaceae and that this association can last for weeks in vitro; suggesting that these amoebae can interact with vibrios in this environment. Although the ecological dynamics of these Vannella-Vibrionaceae associations deserves to be investigated in greater details, our data suggest that the Vannella sp. isolated in the present study are an ecologically relevant host to investigate the biotic interactions between Vibrio species and marine amoebae.

By studying the interactions between Vannella sp. AP1411 and the vibrio LGP32, we found that $V$. tasmaniensis LGP32 is able to resist predation by the marine amoeba. Vibrios belonging to the tasmaniensis species have been shown to resist haemocyte phagocytosis and to behave as facultative intracellular pathogen, a behaviour that was shown to be central for its virulence in oysters (Duperthuy et al., 2011; Vanhove et al., 2016; Rubio et al., 2019). The slow growth of amoebae on LGP32 was due, to some extent, to an inefficient intracellular killing of LGP32. Indeed, after phagocytosis most intracellular LGP32 were isolated in individual phagosomes inside the amoebae without any morphological alteration whereas the non-virulent control $V$. tasmaniensis LMG20012 $^{\top}$ was progressively clustered in vacuoles and degraded. This strongly suggests that LGP32 interferes with phagosome maturation and intracellular trafficking and this is reminiscent of its capacity to inhibit phagosome acidification in oyster phagocytes (Duperthuy et al., 2011).

The copper efflux p-ATPase CopA was found here to play a major role in the capacity of LGP32 to resist intracellular degradation within the amoeba. As CopA is involved in the resistance of LGP32 to high concentration of copper within oyster haemocytes (Vanhove et al., 2016), our results indicate that LGP32 resistance to grazing by the amoeba depends on its capacity to tolerate high concentrations of copper inside phagosomes and thus strongly suggest that Vannella sp. AP1411 use intraphagosomal heavy metal poisoning in a similar manner than other professional phagocytes. Intraphagosomal copper accumulation appears to be an ancestral highly conserved antimicrobial mechanism from FLA, social amoebae (Hao et al., 2016), invertebrates (Vanhove et al., 2016) to vertebrate professional phagocytes (Soldati and Neyrolles, 2012). As this antimicrobial mechanism is used by a variety of phagocytes we can hypothesize that, like other bacterial species resisting intracellular killing (Espariz et al., 2007; Rowland and Niederweis, 2012), for some vibrios, copper resistance confers them a fitness advantage in diverse host-vibrio interactions (Brooks et al., 2014; Vanhove et al., 2016).

Another determinant that was found here to be important for LGP32 resistance to grazing by Vannella sp. AP1411 was the secreted metalloprotease Vsm, a major secreted protease that plays a role as a virulence factor in oysters (Binesse et al., 2008). In the present study, the Vsm deficient LGP32 strain showed an increased sensitivity to grazing by the amoeba, without 
losing its ability to resist intracellular degradation. This suggested that Vsm acts extracellularly rather than intracellularly, during LGP32 interaction with amoeba, which was confirmed by studying amoeba grazing behaviour by time-lapse microscopy revealing an important increase of grazing migratory speed of amoebae on $\Delta v s m$ LGP32 bacteria lawns compared with lawns with the wild-type strain. The involvement of Vsm metalloprotease in LGP32 resistance to grazing is reminiscent of the role played by other proteases secreted by vibrios during their interaction with eukaryotic cells. In particular, the involvement of the secreted protease PrtV, that participates in the resistance of $V$. cholerae to the grazing by the bacteriovorous ciliate Tetrahymena pyriformis (Vaitkevicius et al., 2006), the metalloprotease Vsm can also damage oyster heamocytes (Binesse et al., 2008), and its homologue Vam, from $V$. aestuarianus, can inhibit oyster haemocyte phagocytosis (Labreuche et al., 2010). Interestingly, recent work studying the interaction between clinical isolates of $V$. cholerae and the model amoebae Acanthamoeba castellanii (Neff strain) reported that minor virulence factors like secreted enzymes, including the HapA metalloprotease and the haemolysin, can be involved in fine tuning the fitness of $V$. cholerae during its interaction with grazers in the aquatic environment (Van der Henst et al., 2018). Then the secretion of proteases by vibrios appears to be an important ancestral mechanism that may has been acquired to inhibit predation by grazers in aquatic environments but can also participate to their pathogenicity in animal hosts.

Hence, as copper resistance and secretion of proteases appear to be important virulence mechanisms for different vibrios and involved in interactions with diverse hosts, the presence of these virulence traits in vibrios against diverse hosts may represent evidences for the coincidental selection hypothesis (Adiba et al., 2010).

As bacteriovorous protists exert a selective pressure on bacterial communities, different bacteria species are thought to have developed a diversity of survival strategies against predation through either pre-ingestional or post-ingestional adaptations (Matz and Kjelleberg, 2005). Pre-ingestional adaptations including high motility, filamentation, surface masking or toxin release are commonly found for extracellular pathogens; whereas postingestional adaptations including digestional resistance through vacuolar trafficking inhibition or vacuolar escaping, and toxin release are commonly found for intracellular pathogens and tend to favour bacterial growth (Matz and Kjelleberg, 2005). By establishing a new ecologically relevant cellular model to study the Vibrio-phagocytes interactions in marine environment, we found that, in the case of the facultative intracellular pathogen LGP32, a combination of pre-ingestional and post-ingestional adaptations could be involved. The involvement of the secreted Vsm represents a pre-ingestional adaptation, whereas the involvement of the CopA p-ATPase represents a post-ingestional adaptation. Thus, the predatorprey interactions between bacterial communities and bacterivorous protists may select for common virulence factors allowing bacteria to resist to a diversity of phagocytic cells from amoeba to immune cells in animals, favouring the emergence of opportunistic pathogens able to colonize a diversity of hosts, like many vibrios do.

However, not all virulence factors studied here were found to participate to LGP32 resistance to grazing by Vannella $\mathrm{sp}$. AP1411. For instance, V. tasmaniensis T6SS1, Vsp, CusAB and SOD, which were found to play a role in oysters (Vanhove et al., 2014, 2016; Rubio et al., 2019), do not seem to play a role during interactions with Vannella sp. AP1411. These results strengthen the hypothesis that although some virulence factors could be involved in diverse biotic interactions, as observed here for CopA and Vsm, hence favouring opportunism, some other virulence factors could be more specifically involved in more specialized interactions. As for example, the metalloprotease InhA, the orthologue of PrtV in LGP32, does not appear to play a role during LGP32-Oyster interactions or LGP32-Vannella sp. AP1411 interaction, although it was shown to participate in the resistance of $V$. cholerae to the grazing by the bacteriovorous ciliate Tetrahymena pyriformis (Vaitkevicius et al., 2006). In a similar manner, T6SS, which was shown to be involved during interaction of Vibrio cholerae with the social amoeba $D$. discoideum (Pukatzki et al., 2006), did not appear to be involved in LGP32 resistance to Vannella sp. AP1411 although it was shown recently to be a major determinant of LGP32 cytotoxic activity towards oyster haemocytes (Rubio et al., 2019). Such host-specialization of some vibrios virulence factors was also suggested recently for $V$. cholerae, as some of the virulence factors involved in the resistance to grazing by $A$. castellanii are considered to have a minor role in pathogenesis in human (Van der Henst et al., 2018). These subtleties in the involvement of different virulence factors during different biotic interactions are reminiscent of the subtleties that are being uncovered for the intracellular pathogen $L$. pneumophila. This well studied intracellular pathogen harbours a broad range of virulence factors and can interact with a diversity of hosts, but this does not imply that all virulence factors have a similar importance in each interactions with these diverse hosts (Boamah et al., 2017; Ghosh and O'Connor, 2017). Vibrios being mostly opportunistic pathogens, they probably have acquired, on the one hand, some virulence factors that are involved in diverse host interactions and could represent core virulence factors, and on the other hand, some virulence factors that are involved in more restricted host interactions that could represent specialized virulence factors. Studying diverse host-vibrios interactions will help to decipher the diversity of their virulence factors and provide insights about opportunist pathogens co-evolutionary trajectories that are 
more complex than the host-parasite co-evolutionary arm race observed for host specialized pathogens (Brown et al., 2012).

In conclusion, by developing a new ecologically relevant cellular model of interaction, we showed here that the oyster pathogen $V$. tasmaniensis LGP32 can resist phagocytosis by environmental grazers Vannella spp., which are ubiquitous in oyster farming area and appear to interact with vibrios in the oyster farming environment. By performing comparative cellular biology, we also demonstrated that some of LGP32 virulence factors can be involved in different types of interactions with multiple hosts from protozoans, such as environmental marine amoeba, to metazoans, for example, oysters. This suggests that some virulence factors such as CopA and Vsm could play a central role in the interaction of $V$. tasmaniensis with diverse hosts whereas some other virulence factors appear to be involved in more specific host-vibrio interactions. Hence, the present study implies that studying diverse host-vibrio interactions will help to gain further insights about the ecology of vibrios and to uncover the relative roles of biotic interactions in pathogen emergence.

\section{Materials and methods}

\section{Bacterial strains and growth conditions}

Escherichia coli strain SBS363 was grown in Luria-Bertani (LB) or LB-agar (LBA) at $37^{\circ} \mathrm{C}$. Marine amoebae Vannella sp. AP1411 and isolates 0517-TOEc-2, 0517-TOEc-3, 0517-TOEc-4, 0517-BWEc-1, 0517-BWEc-2, 0517-BWEc-3, 0517-BWEC-4 (isolated in this study) were grown in 70\% sterile seawater (SSW) with E. coli wild-type strain SBS363 at $18^{\circ} \mathrm{C}$, for 3 days prior experiments. Vibrios strains used in this study are V. tasmaniensis LMG20012 ${ }^{\top}$, V. tasmaniensis LGP32 and previously described LGP32 deletion mutants $\Delta$ copA, $\Delta$ cusAB; $\Delta$ sodA; $\Delta v s m, \Delta v s p, \Delta i n h A$ (Binesse et al., 2008; Vanhove et al., 2014, 2016) and insertion mutants $\Delta$ vipA1, $\Delta$ vipA2 (Rubio et al., 2019). Vibrios strains carrying the pMRB-GFP or pD3-GFP-mut3 plasmids were grown in $\mathrm{LB}+\mathrm{NaCl} 0.5 \mathrm{M}$ supplemented with chloramphenicol $\left(10 \mu \mathrm{g} \mathrm{mL}^{-1}\right)$ or kanamycin $\left(20 \mu \mathrm{g} \mathrm{mL}^{-1}\right)$ at $20^{\circ} \mathrm{C}$, for $24 \mathrm{~h}$ prior experiments.

\section{Isolation of environmental amoebae from the Thau Lagoon and Banyuls-sur-mer}

Between 2014 and 2015, seawater, oysters and sediment from the Thau lagoon (South France) were sampled at least two times per season. Seawater was collected next to oyster tables at the Bouzigues station IfremerREPHY (GPS: N 43 $26.058^{\prime}$ E $03^{\circ} 39^{\prime} 0.878^{\prime}$ ) and filtered, on the boat, with a $180 \mu \mathrm{m}$ pore size nylon filter. In the lab, the water was re-filtered using an $8.0 \mu \mathrm{m}$ pore size MF-Millipore membrane. The $8.0 \mu \mathrm{m}$ pore size membrane was then cut in four pieces and each quarter was put upside down on a lawn of $E$. coli SBS363 seeded on SSW-agar. Oyster gills were cut in four pieces of roughly $0.5 \mathrm{~cm}^{2}$ and each quarter was put upside down on a lawn of $E$. coli SBS363 seeded on non-nutrient SSW-agar. Sediment was collected under the oyster tables (depth of $9 \mathrm{~m}$ ) by core sampling. One gram of sediment was then deposited in the centre of a lawn of E. coli SBS363 seeded on SSW-agar, in triplicate. After 1-3 weeks, depending of the sampling season, migrating amoebae were observed at the periphery of the plates. An agar square of $0.5 \times 0.5 \mathrm{~cm}$ containing a single amoeba was then cut and deposited upside down on a fresh lawn of $E$. coli SBS363 seeded on SSW-agar. This was repeated several times to isolate and replicate amoebae. DNA was extracted using the High Pure PCR Template Preparation Kit (Roche), according to the manufacturers' protocol. In May 2017, sampling was performed from oyster gills from Thau lagoon at Bouzigues station Ifremer-REPHY (GPS: N 43 $26.058^{\prime}$ E $03^{\circ} 39^{\prime} 0.878^{\prime}$ ) and seawater from Banyuls-sur-mer at SOLA station (GPS: $42^{\circ} 29^{\prime} 300 \mathrm{~N} \mathrm{-}$ $03^{\circ} 08^{\prime} 700 \mathrm{E}$ ). Samples were treated with the same protocol than previously and put on a lawn of $E$. coli SBS363 seeded on SSW-agar. After 2 weeks, amoebae were flushed by SSW and clonally isolated by dilutions within 96 well plates with statistically less than one amoeba per well. Amoebae clones were amplified by culturing in six well plates, and DNA was extracted using the NucleoSpin Tissue kit (Macherey-Nagel), according to the manufacturer's protocol.

\section{Identification of isolated marine amoebae from Thau Lagoon and Banyuls-sur-Mer and their associated bacteria}

In order to infer the phylogenetic assignment of environmental amoebae, the v7 region of the 18s rDNA gene was sequenced using specific primers. The primers were designed using SILVA database dedicated tool (http://www. arb-silva.de/search/testprime). The selected primers, Amo_AP_1154_F: 5'GAGRAAATTAGAGTGTTYAAAG $3^{\prime}$ and Amo_AP_1470_R: 5'TTATRGTTAAGACTA CGACGG $3^{\prime}$, were used to amplify the hypervariable region V7 of $18 \mathrm{~S}$ rDNA gene at an annealing temperature of $54^{\circ} \mathrm{C}$. PCR amplicons were cloned using the TOPO TA Cloning kit (Invitrogen), according to the manufacturers' protocol. Nucleotide sequences were determined by Sanger sequencing (GenSeq platform, Labex CEMEB, Montpellier, France). Sequence homologies were searched using BLASTn available at the NCBI website (National Centre for Biotechnology Information) (Wheeler et al., 2007) (Table S1). For phylogenetic analyses, sequences were aligned in MOTHUR 
(Schloss, 2009) and imported into ARB software (Ludwig et al., 2004) loaded with the silva database (http://www.arbsilva.de). A base frequency filter was applied to exclude highly variable positions before adding sequences to the maximum parsimony backbone tree using the parsimony quick add marked tool implemented in ARB, thereby maintaining the overall tree topology provided by default. In order to verify that the Sanger sequencing strategy used here allowed us to identify the entire diversity of amoebae that could be isolated through culturing technics. The total DNA from our different amoebae cultures was analysed by performing barcoding on the $\mathrm{v} 7$ region of the 18S rDNA gene using generic primers that allowed amplifying more than $80 \%$ of all the eukaryote 18s rDNA sequences available in SILVA database (F-v7-1173: CCT GCG GCT TAA TTT GAC and Rv7-1438: CAT CAC AGA CCT GTT ATT GC). Sequencing was performed by Genome Quebec facility, Montreal, Canada.

Bacteria associated with amoeba clonally isolated in May 2017 sampling were investigated using barcoding sequencing on the V3/V4 region of the 16S rDNA gene with Illumina generic primers according to the manufacturer's protocol (16Sv3v4-ILLAFWD: 5'-TCG TCG GCA GCG TCA GAT GTG TAT AAG AGA CAG YRC CTA CGG GNG GCW GCAG and 16Sv3v4-ILLAREV: 5'-GTC TCG TGG GCT CGG AGA TGT GTA TAA GAG ACA GYR GAC TAC HVG GGT ATC TAA TCC). Sequencing was performed by GenSeq platform, Labex CEMEB, Montpellier, France. OTUs taxonomic affiliation was performed using FROGS tool from galaxy software (Escudié et al., 2018). To determine the taxonomic affiliation of clones isolated during the second sampling campaign, the sequence of the V4 hypervariable region of the 18S rDNA gene was analysed using TAReuk454FWD1: 5'-CCAGCA(G/C)C(C/T)GCGGTAATTCC-3' and TAReukREV3: 5'-ACTTTCGTTCTTGAT (C/T)(A/G)A-3' primers (Stoeck et al., 2010). Nucleotide sequences were determined by Sanger sequencing (GenSeq platform, Labex CEMEB, Montpellier, France) and aligned with MUSCLE using MEGA7 software (maximumlikelihood by bootstrap method, 1000 replications).

\section{Grazing assay}

To prepare the co-culture of vibrios and amoebae, $1 \mathrm{ml}$ of vibrio overnight culture $\left(3.10^{9}\right.$ bacteria. $\left.\mathrm{mL}^{-1}\right)$ was mixed with $100 \mu$ l of 3 days old Vannella sp. AP1411 culture $\left(5 \times 10^{5}\right.$ cells $\left.\mathrm{mL}^{-1}\right)$ or with $100 \mu$ of $70 \%$ SSW for control condition. A volume of $50 \mu \mathrm{l}$ per well of the mixed culture was seeded on top of $500 \mu \mathrm{l}$ of $1 \% \mathrm{SSW}$-agar, in 24-well plates with transparent flat bottoms. Amoebae and bacteria lawns were carefully homogenized in the wells and let dry $4 \mathrm{~h}$ at room temperature in a laminar flow cabinet and then incubated at $18^{\circ} \mathrm{C}$ in a humidified atmosphere. GFP fluorescence intensity was measured every day over 7 days using a TECAN plate reader ( $\lambda$ ex $480 \mathrm{~nm} / \lambda \mathrm{em} 520 \mathrm{~nm}$ ). To estimate the effect of the amoebae grazing activity on the abundance of living vibrios expressing GFP, the fluorescence intensity of the wells containing amoebae was compared with the fluorescence of vibrios lawn without amoebae, and expressed as a ratio. Each condition was performed in technical triplicates and the results shown are the average of three independent experiments. Error bars represent the standard error of the mean $( \pm S E M)$. Statistical analysis was performed using RM-ANOVA over the independent experiments.

\section{Monitoring amoeba growth and association with GFP- vibrios}

To estimate the proliferation of Vannella sp. AP1411 at days 1,3 and 6 in grazing assays, amoebae were directly imaged by phase-contrast microscopy and enumerated in triplicate for each condition. Cells were flushed from the SSW-agar surface with $1 \mathrm{ml}$ of $70 \%$ SSW and fixed for 30 min with $2 \%$ paraformaldehyde at room temperature. Pellets were washed and suspended in $500 \mu$ l of PBS for flow cytometry analysis (BD FACS Canto). Data analyses were performed using Flowing Software by gating on amoeba cells using SSC/FSC parameters then quantifying the percentage of GFP fluorescence associated amoebae (as shown in Fig. S2). In order to estimate the phagocytosis index, which corresponds to the average number of phagocytosed bacteria per cell, the mean intensity of GFP fluorescence per amoeba was normalized to the mean GFP fluorescence per bacteria (as shown in Fig. S3). The experiments were performed with three technical replicates per condition in each experiment, and depicted results are representative of at least two independent experiments. Error bars represent the \pm SEM. Statistical analysis was performed using two ways ANOVA with HolmSidak's multiple comparisons test.

\section{Fluorescence in situ hybridization}

In situ hybridization for Vibrionaceae was performed on Vannella sp. AP141, 0517-TOEc-2, 0517-TOEc-3 and 0517-TOEc-4. After at least 2 weeks of culturing in flask in $70 \%$ SSW complemented with E. coli SBS363, amoebae were washed twice with $3 \mathrm{ml}$ of $70 \%$ SSW and resuspended in $70 \%$ SSW using a cell scrapper and fixed with $2 \%$ formaldehyde for $25 \mathrm{~min}$ at room temperature. After fixation, formaldehyde was removed by centrifugation of the cells by centrifugation $10 \mathrm{~min}$ at $6000 \mathrm{~g}$, pellets were washed and resuspended in $500 \mu$ of PBS. Cell suspensions were cytospun on glass slides for $10 \mathrm{~min}$ at $500 \mathrm{~g}$. Glass slides were then labelled with the 
Vibrionaceae specific probe VIB572a at $10 \mathrm{ng} \mathrm{mL}^{-1}\left(5^{\prime}\right.$ ACCACCTGCATGCGCTTT-3') (Huggett et al., 2008) using hybridization buffer $(900 \mathrm{mM} \mathrm{NaCl}, 20 \mathrm{mM}$ Tris$\mathrm{HCl}, 30 \%$ Formamide, $0.01 \% \mathrm{SDS}, \mathrm{dH}_{2} \mathrm{O}$ ) for $90 \mathrm{~min}$ at $46^{\circ} \mathrm{C}$ in the dark. The samples were then washed for $25 \mathrm{~min}$ in washing buffer $(0.112 \mathrm{mM} \mathrm{NaCl}, 20 \mathrm{mM}$ Tris$\mathrm{HCl}, 0.01 \%$ SDS, $5 \mathrm{mM}$ EDTA, dH2O) at $48^{\circ} \mathrm{C}$ in the dark. Then, cells were counterstained using FITC at $0.5 \mathrm{ng} \mathrm{mL}^{-1}$ for $30 \mathrm{~min}$ (Sigma). Confocal fluorescence imaging was performed using a $63 \times$ oil objective and images were captured using a Leica TCS SPE confocal scanning laser microscope, at 1 airy to ensure the focal plan.

\section{Microscopy}

The different amoebae shapes were observed on living amoebae using phase contrast (on an inverted wide-field microscope, Zeiss). The shape of the floating forms was recorded by scraping in the cell suspension followed by immediate observation. The cystic form was observed after amoebae were starved for a week. Intracellular organization of amoebae was observed using transmission electron microscopy. Briefly, amoebae cultured in flasks were washed twice using $70 \%$ SSW, and fixed in $2.5 \%$ glutaraldehyde for $2 \mathrm{~h}$ in the dark at room temperature, followed by overnight at $4^{\circ} \mathrm{C}$. After centrifugation, the supernatant was replaced by $3 \%$ low melting-point agarose (Sigma) and the pellet was resuspended. Cells entrapped in $3 \%$ agar were immersed in a solution of $2.5 \%$ glutaraldehyde in PHEM buffer $(1 \times, \mathrm{pH} 7.4)$ overnight at $4^{\circ} \mathrm{C}$. Then they were rinsed in PHEM buffer and post-fixed in a $0.5 \%$ osmic acid for $2 \mathrm{~h}$ in the dark at room temperature. After two rinses, samples were dehydrated in a graded series of ethanol solutions $(30 \%-$ 100\%). Samples were embedded in EmBed 812 using an Automated Microwave Tissue Processor for Electronic Microscopy, Leica EM AMW. Ultrathin sections $(70 \mathrm{~nm}$; Leica-Reichert Ultracut E) were collected at different levels of each block, then counterstained with uranyl acetate $1.5 \%$ in $70 \%$ ethanol and lead citrate and observed using a Tecnai F20 transmission electron microscope at $120 \mathrm{kV}$ in the CoMET MRI facilities, INM, Montpellier, France.

Confocal fluorescence microscopy was performed on amoebae suspensions after PFA fixation to observe the localization of intra-amoeba vibrios. Cell suspensions were cytospun on glass slides for $10 \mathrm{~min}$ at $800 \mathrm{~g}$. Glass slides were then stained with $0.25 \mu \mathrm{g} \mathrm{mL}^{-1}$ DAPI (Sigma) and $0.1 \mu \mathrm{g} \mathrm{m}^{-1}$ Blue Evans (Sigma). Confocal fluorescence imaging was performed using a $63 \times$ oil objective and images were captured using a Leica TCS SPE confocal scanning laser microscope, at 1 airy to ensure the focal plan.

\section{Time-lapse imaging and cell tracking}

Co-cultures of vibrio and amoebae were prepared following the same protocol as for grazing assays. Time-lapse imaging started $24 \mathrm{~h}$ after the beginning of the grazing assays. Acquisitions were obtained using an inverted wide-field epifluorescence microscope (Zeiss) and B\&W coolsnap camera. Time-lapses were done during $30 \mathrm{~min}$ with one frame every $30 \mathrm{~s}$. TrackMate Tools software (ImageJ) was used for manual tracking to determine velocity and migration distance by amoebae on different vibrios lawns (Supplementary Movies 1, 2 and 3). Migration distances were measured for 18 amoebae at the same time (i.e. 20 frames per amoebae trace) for each condition. Each track depicted corresponds to the migration of an amoeba during $10 \mathrm{~min}$. The migration path was determined by the $x$ and $y$ positions of amoebae tracked at each frame. Depicted results are representative of at least two independent experiments. Velocities were measured for 16 amoebae tracks, only tracks made of at least five frames were considered. Each presented result corresponds to the average velocity of one amoeba per track. Depicted results are representative of at least two independent experiments. Error bars represent the standard deviation $( \pm S D)$. Statistical analysis was performed using Kruskal-Wallis test with Dunn's multiple comparisons test.

\section{Acknowledgements}

We are grateful to Thibaut Groult, Audrey Caro, and Marc Leroy for precious help in sample collection and preparation, to Eric Abadie for field trip coordination and to Michel Cantou from the University of Montpellier for scuba diving allowing sediment collection. We thank Marie Buysse and Olivier Duron for technical advices. This work, through the use of the GENSEQ platform (http://www.labex-cemeb.org/ fr/genomique-environnementale-2) from the labEx CeMEB. The authors also thank the Montpellier RIO imaging platform (https://www.mri.cnrs.fr). The present study was supported by the EU funded project VIVALDI (H2020 program, No. 678589), by the Ec2co-CNRS funded Intervibrio and VibrAm projects, by Labex CEMEB Amibadapt project, and by Ifremer, University of Montpellier and University of Perpignan via Domitia.

\section{Conflict of Interest}

The authors declare that there are no conflict of interests related to this work.

\section{References}

Adiba, S., Nizak, C., van Baalen, M., Denamur, E., and Depaulis, F. (2010) From grazing resistance to pathogenesis: the coincidental evolution of virulence factors. PLoS One 5: 1-10. 
Austin, B. (2010) Vibrios as causal agents of zoonoses. Vet Microbiol 140: 310-317.

Binesse, J., Delsert, C., Saulnier, D., ChampomierVergès, M.-C., Zagorec, M., Munier-Lehmann, H., et al. (2008) Metalloprotease vsm is the major determinant of toxicity for extracellular products of Vibrio splendidus. Appl Environ Microbiol 74: 7108-7117.

Boamah, D.K., Zhou, G., Ensminger, A.W., and O'Connor, T.J. (2017) From many hosts, one accidental pathogen: the diverse protozoan hosts of legionella. Front Cell Infect Microbiol 7: 477.

Boulais, J., Trost, M., Landry, C.R., Dieckmann, R., Levy, E. D., Soldati, T., et al. (2010) Molecular characterization of the evolution of phagosomes. Mol Syst Biol 6: 423.

Brooks, J.F., Gyllborg, M.C., Cronin, D.C., Quillin, S.J., Mallama, C.A., Foxall, R., et al. (2014) Global discovery of colonization determinants in the squid symbiont Vibrio fischeri. Proc Natl Acad Sci $U S A$ 111: 17284-17289.

Brown, S.P., Cornforth, D.M., and Mideo, N. (2012) Evolution of virulence in opportunistic pathogens: generalism, plasticity, and control. Trends Microbiol 20: 336-342.

Bruto, M., James, A., Petton, B., Labreuche, Y., Chenivesse, S., Alunno-Bruscia, M., et al. (2017) Vibrio crassostreae, a benign oyster colonizer turned into a pathogen after plasmid acquisition. ISME $J$ 11: 1043-1052.

Constantin de Magny, G., Murtugudde, R., Sapiano, M.R.P., Nizam, A., Brown, C.W., Busalacchi, A.J., et al. (2008) Environmental signatures associated with cholera epidemics. Proc Natl Acad Sci U S A 105: 17676-17681.

de Lorgeril, J., Lucasson, A., Petton, B., Toulza, E., Montagnani, C., Clerissi, C., et al. (2018) Immunesuppression by OsHV-1 viral infection causes fatal bacteraemia in Pacific oysters. Nat Commun 9: 4215.

de Souza Santos, M., and Orth, K. (2014) Intracellular Vibrio parahaemolyticus escapes the vacuole and establishes a replicative niche in the cytosol of epithelial cells. MBio 5: e01506-e01514.

Duperthuy, M., Schmitt, P., Garzón, E., Caro, A., Rosa, R. D., Le Roux, F., et al. (2011) Use of OmpU porins for attachment and invasion of Crassostrea gigas immune cells by the oyster pathogen Vibrio splendidus. Proc Natl Acad Sci U S A 108: 2993-2998.

Escudié, F., Auer, L., Bernard, M., Mariadassou, M., Cauquil, L., Vidal, K., et al. (2018) FROGS: find, rapidly, OTUs with galaxy solution. Bioinformatics 34: 1287-1294.

Espariz, M., Checa, S.K., Audero, M.E.P., Pontel, L.B., and Soncini, F.C. (2007) Dissecting the Salmonella response to copper. Microbiology 153: 2989-2997.

Gay, M., Renault, T., Pons, A., and Le Roux, F. (2004) Two Vibrio splendidus related strains collaborate to kill Crassostrea gigas: taxonomy and host alterations. Dis Aquat Organ 62: 65-74.

Ghosh, S., and O'Connor, T.J. (2017) Beyond paralogs: the multiple layers of redundancy in bacterial pathogenesis. Front Cell Infect Microbiol 7: 1-14.

Greub, G., and Raoult, D. (2004) Microorganisms resistant to free-living amoebae. Clin Microbiol Rev 17: 413-433.

Hao, X., Luthje, F., Rønn, R., German, N.A., Li, X., et al. (2016) A role for copper in protozoan grazing - two billion years selecting for bacterial copper resistance. Mol Microbiol 102: 628-641.

Huggett, M., Crocetti, G., Kjelleberg, S., and Steinberg, P. (2008) Recruitment of the sea urchin Heliocidaris erythrogramma and the distribution and abundance of inducing bacteria in the field. Aquat Microb Ecol 53: 161-171.

Labreuche, Y., Le Roux, F., Henry, J., Zatylny, C., Huvet, A., Lambert, C., et al. (2010) Vibrio aestuarianus zinc metalloprotease causes lethality in the Pacific oyster Crassostrea gigas and impairs the host cellular immune defenses. Fish Shellfish Immunol 29: 753-758.

Le Roux, F., Wegner, K.M., Baker-Austin, C., Vezzulli, L., Osorio, C.R., Amaro, C., et al. (2015) The emergence of Vibrio pathogens in Europe: ecology, evolution, and pathogenesis (Paris, 11-12th March 2015). Front Microbiol 6: 830.

Lemire, A., Goudenège, D., Versigny, T., Petton, B., Calteau, A., Labreuche, Y., and Le Roux, F. (2014) Populations, not clones, are the unit of vibrio pathogenesis in naturally infected oysters. ISME J 9: 1523-1531.

Lopez-Joven, C., Rolland, J.L., Haffner, P., Caro, A., Roques, C., Carré, C., et al. (2018) Oyster farming, temperature, and plankton influence the dynamics of pathogenic Vibrios in the Thau Lagoon. Front Microbiol 9: 2530.

Ludwig, W., Strunk, O., Westram, R., Richter, L., Meier, H., Yadhukumar, et al. (2004) ARB: a software environment for sequence data. Nucleic Acids Res 32: 1363-1371.

Ma, A.T., McAuley, S., Pukatzki, S., and Mekalanos, J.J. (2009) Translocation of a Vibrio cholerae type VI secretion effector requires bacterial endocytosis by host cells. Cell Host Microbe 5: 234-243.

Matz, C., and Kjelleberg, S. (2005) Off the hook - how bacteria survive protozoan grazing. Trends Microbiol 13: 302-307.

Molmeret, M., Horn, M., Wagner, M., Santic, M., and Abu Kwaik, Y. (2005) Amoebae as training grounds for intracellular bacterial pathogens. Appl Environ Microbiol 71: 20-28.

Pernthaler, J. (2005) Predation on prokaryotes in the water column and its ecological implications. Nat Rev Microbiol 3: $537-546$.

Pukatzki, S., Ma, A.T., Sturtevant, D., Krastins, B., Sarracino, D., Nelson, W.C., et al. (2006) Identification of a conserved bacterial protein secretion system in Vibrio cholerae using the Dictyostelium host model system. Proc Natl Acad Sci U S A 103: 1528-1533.

Ritchie, J.M., Rui, H., Zhou, X., lida, T., Kodoma, T., Ito, S., et al. (2012) Inflammation and disintegration of intestinal villi in an experimental model for Vibrio parahaemolyticusinduced Diarrhea. PLoS Pathog 8: e1002593.

Rosenberg, E., and Falkovitz, L. (2004) The Vibrio shiloi/Oculina patagonica model system of coral bleaching. Annu Rev Microbiol 58: 143.

Rowbotham, T.J. (1980) Preliminary report on the pathogenicity of Legionella pneumophila for freshwater and soilamoebae. J Clin Pathol 33: 1179-1183.

Rowland, J.L., and Niederweis, M. (2012) Resistance mechanisms of Mycobacterium tuberculosis against phagosomal copper overload. Tuberculosis 92: 202-210.

Rubio, T.P., Oyanedel, D., Labreuche, Y., Toulza, E., Luo, X., Bruto, M., et al. (2019) Species-specific mechanisms of cytotoxicity toward immune cells determine the 
successful outcome of Vibrio infections. Proc Natl Acad Sci U S A 116: 14238-14247.

Schloss, P.D. (2009) A high-throughput DNA sequence aligner for microbial ecology studies. PLoS One 4: e8230.

Smirnov, A.V., Nassonova, E.S., Chao, E., and CavalierSmith, T. (2007) Phylogeny, evolution, and taxonomy of Vannellid amoebae. Protist 158: 295-324.

Soldati, T., and Neyrolles, O. (2012) Mycobacteria and the intraphagosomal environment: take it with a pinch of salt(s)! Traffic 13: 1042-1052.

Stoeck, T., Bass, D., Nebel, M., Christen, R., Jones, M.D.M., Breiner, H.W., and Richards, T.A. (2010) Multiple marker parallel tag environmental DNA sequencing reveals a highly complex eukaryotic community in marine anoxic water. Mol Ecol 19: 21-31.

Takemura, A.F., Chien, D.M., and Polz, M.F. (2014) Associations and dynamics of Vibrionaceae in the environment, from the genus to the population level. Front Microbiol 5: 38 .

Vaitkevicius, K., Lindmark, B., Ou, G., Song, T., Toma, C., Iwanaga, M., et al. (2006) A Vibrio cholerae protease needed for killing of Caenorhabditis elegans has a role in protection from natural predator grazing. Proc Natl Acad Sci U S A 103: 9280-9285.

Van der Henst, C., Vanhove, A.S., Drebes Dörr, N.C., Stutzmann, S., Stoudmann, C., Clerc, S., et al. (2018) Molecular insights into Vibrio cholerae's intra-amoebal host-pathogen interactions. Nat Commun 9: 3460.

Vanhove, A.S., Duperthuy, M., Charrière, G.M., Le Roux, F., Goudenège, D., Gourbal, B., et al. (2014) Outer membrane vesicles are vehicles for the delivery of Vibrio tasmaniensis virulence factors to oyster immune cells. Environ Microbiol 17: 1152-1165.

Vanhove, A.S., Rubio, T.P., Nguyen, A.N., Lemire, A., Roche, D., Nicod, J., et al. (2016) Copper homeostasis at the host vibrio interface: lessons from intracellular vibrio transcriptomics. Environ Microbiol 18: 875-888.

Vidal-Dupiol, J., Ladriere, O., Meistertzheim, A.-L., Foure, L., Adjeroud, M., and Mitta, G. (2011) Physiological responses of the scleractinian coral Pocillopora damicornis to bacterial stress from Vibrio coralliilyticus. J Exp Biol 214: 1533-1545.

Wheeler, D.L., Barrett, T., Benson, D.A., Bryant, S.H., Canese, K., Chetvernin, V., et al. (2007) Database resources of the National Center for biotechnology information. Nucleic Acids Res 36: D13-D21.

\section{Supporting Information}

Additional Supporting Information may be found in the online version of this article at the publisher's web-site:

Fig. S1. Phylogeny of the diversity of amoebae OTUs found by barcoding of the $v 7$ region of the $18 \mathrm{~s}$ rDNA gene. Phylogenetic 18S rRNA-based trees of the Amoebozoa constructed with ARB software (http://www.arb- home.de (Ludwig et al., 2004)) loaded with the silva database (http://www.arb-silva.de). A base frequency filter was applied using the parsimony quick add marked tool implemented in ARB. All 18S rDNA gene sequences assigned to amoeba belonged to the Vannellidae family (written in red).

Fig. S2. Vannella sp. AP1411 isolated from Thau lagoon, France.

(A) Different forms of Vannella sp. AP1411 under phase light microscopy. Trophozoite (left), pelagic (centre) and cystic form (right). Scale bar: $10 \mu \mathrm{m}$. (B) Transmission electron microscopy of trophozoite shape of the amoeba Vannella $\mathrm{sp}$. AP1411. Whole cell overview showing nuclei (N) and several digestive vacuoles (DV) with or without $E$. coli SBS363 bacteria inside (B). Scale bar: $2 \mu \mathrm{m}$.

Fig. S3. Quantification of Vannella sp. AP1411 phagocytosis by cytometry. (A) FSC/SSC Gate corresponding to amoebae (B) Determination of fluorescence background on amoebae alone. (C) Gate determination for GFP+ vibrios (Bacteria). (D) Measurement of the percentage of amoebae carrying GFP fluorescence after 3 days of co-culture with GFP-expressing vibrios.

Fig. S4 CusAB, SodA, Vsp, InhA, VipA1 and VipA2 are not involved in LGP32 resistance to grazing by Vannella sp. 1411.

Bacterial resistance to grazing by Vannella sp. AP1411 of $\triangle$ cusAB, $\Delta$ sodA, $\Delta v s p, \triangle i n h A, \Delta v i p A 1$ and $\Delta v i p A 2$ mutant strains of LGP32 was assessed by measuring the fluorescence of the GFP-expressing bacteria, after contact with amoebae. Each condition was performed in triplicate and the results shown are the average of three independent experiments. Values are presented \pm SEM.

Fig. S5. Vsm or CopA do not affect the phagocytosis rate of LGP32 by the amoeba Vannella sp. AP1411. Grazing amoebae on GFP-vibrio LGP32wt, LGP32 $\Delta$ copA or of LGP32 $\Delta v s m$ were monitored by flow cytometry to estimate phagocytosis index per amoeba, which correspond to the average number of phagocyted bacteria per cell. The results shown are representative of two independent experiments. Data were analysed by one-way ANOVA with Friedman test.

Table S1. Sanger sequences of sampled marine amoebae Supplementary Movie 1. Time-lapse microscopy movie of Vannella sp. AP1411 and LGP32wt lawn in co-culture.

Supplementary Movie 2. Time-lapse microscopy of Vannella sp. AP1411 and LGP32 $\triangle$ copA lawn in coculture.

Supplementary Movie 3. Time-lapse microscopy of Vannella sp. AP1411 and LGP32 $\Delta v s m$ lawn in coculture. 